\title{
Representation theorems for stop and play models with input- dependent shape functions
}

\author{
AUTHOR(S): \\ Matsuo, T; Shimasaki, M
}

\section{CITATION:}

Matsuo, $T$...[et al]. Representation theorems for stop and play models with in putdependent shape functions. IEEE TRANSACTIONS ON MAGNETICS 2005, 41(5): 1548-1551

\section{ISSUE DATE:}

2005-05

URL:

http://hdl.handle.net/2433/40003

\section{RIGHT:}

(c)2005 IEEE. Personal use of this material is permitted. However, permission to reprint/republish this material for advertising or promotional purposes or for creating new collective works for resale or redistribution to servers or lists, or to reuse any copyrighted component of this work in other works must be obtained from the IEEE. 


\title{
Representation Theorems for Stop and Play Models With Input-Dependent Shape Functions
}

\author{
Tetsuji Matsuo, Member, IEEE, and Masaaki Shimasaki, Member, IEEE \\ Department of Electrical Engineering, Graduate School of Engineering, Kyoto University, Kyoto 615-8510, Japan
}

\begin{abstract}
Stop and play models with input-dependent shape functions are equivalent to the nonlinear Preisach model proposed by Mayergoyz. This equivalence engenders the necessary and sufficient conditions for a hysteretic nonlinearity to be represented by stop and play models that have input-dependent shape functions. A representation theorem for the stop model having an input-independent shape function is also given. The latter theorem is used to improve the representation accuracy of the stop model for a silicon steel sheet.
\end{abstract}

Index Terms-Equal vertical chords, hysteresis, input-dependent shape function, silicon steel sheet, stop and play models.

\section{INTRODUCTION}

$\mathbf{F}$ INITE-ELEMENT analyses of hysteretic magnetic fields require an efficient hysteresis model. For example, play and stop models [1]-[3] can be implemented more simply than the Preisach model [4]. Moreover, they can describe complex hysteretic phenomena as precisely as the Preisach model.

The stop model can be an efficient tool for magnetic-field analysis because it can provide a hysteretic output of $H$ directly from an input of $B$. However, a previous study [7] has shown that the stop model cannot represent the hysteretic characteristics of a silicon steel sheet sufficiently because the magnetic characteristics of the silicon steel sheet do not satisfy the property of equal vertical chords regardless of dc bias [5], [6]. That property is required for representation by the stop model. Another study [8] has proposed an input-dependent shape function for the stop model to remove this property. That study showed that the input-dependent shape function improves the representation capability of the stop model effectively.

On the other hand, the play model with input-independent shape function has been proven to be equivalent to the scalar static Preisach model [3], [9]. A previous comparison [7] showed that the play model with input-independent shape function can simulate $B-H$ loops of a silicon steel sheet more accurately than the stop model with input-independent shape function. Play and stop models having input-dependent shape functions should be mutually compared because the use of the input-dependent shape function is expected to enhance the representation capability of the play model.

This paper first shows the equivalence of the play and stop models with input-dependent shape functions to the nonlinear Preisach model proposed by Mayergoyz. The equivalence leads to a representation theorem for the stop and play models with input-dependent shape functions. Next, the paper gives a representation theorem for the stop model having an input-independent shape function. That theorem is used to reduce the representation error of the stop model.

\section{Representation Theorems for Stop ANd Play Models}

\section{A. Play Model With Input-Dependent Shape Function}

A play model [1]-[3] having an input-dependent shape function is given as

$$
P(x)=\int_{0}^{x \mathrm{~s}} f_{\zeta}\left(p_{\zeta}(x), x\right) \mathrm{d} \zeta
$$

where $p_{\zeta}$ is the play hysteron operator, $f_{\zeta}$ is the input-dependent shape function for $p_{\zeta}$, and $\zeta$ is a nonnegative parameter that gives the width of the play hysteron. $P(x)$ is assumed to exhibit its hysteretic property only when $|x|<x_{\mathrm{S}}$, where $x_{\mathrm{S}}$ is a positive constant.

The play hysteron operator of ordinary type is given by

$$
p_{\zeta}(x)=\max \left(\min \left(p_{\zeta}^{0}, x+\zeta\right), x-\zeta\right)
$$

where $p_{\zeta}^{0}$ is the value of $p_{\zeta}$ at the previous time-point. However, the function $P(x)$, given by (1) and (2), has a hysteretic property even when $|x| \geq x_{\mathrm{S}}$.

In order for $P(x)$ to become a single-valued function when $|x| \geq x_{\mathrm{S}}$, the operator $p_{\zeta}(x)$ is defined as the following:

$$
\begin{aligned}
p_{\zeta}(x) & =\left\{\begin{array}{cc}
\max \left(\min \left(p_{\zeta}^{0 *}, x+\zeta\right), x-\zeta\right) & \left(0 \leq \zeta<x_{\mathrm{S}}\right) \\
0 & \left(\zeta=x_{\mathrm{S}}\right)
\end{array}\right. \\
p_{\zeta}^{0 *} & =\max \left(\min \left(p_{\zeta}^{0}, x_{\mathrm{S}}-\zeta\right),-x_{\mathrm{S}}+\zeta\right) .
\end{aligned}
$$

Fig. 1(a) illustrates characteristics of this operator for $\zeta<x_{\mathrm{S}}$.

By defining $\nu(\zeta, p, x)=\partial f_{\zeta}(p, x) / \partial p, P(x)$ is written as

$$
\begin{aligned}
P(x) & =P_{M}^{+}(x)+\int_{0}^{x_{S}} \int_{\bar{p}_{+}(\zeta, x)}^{p_{\zeta}(x)} \nu(\zeta, p, x) \mathrm{d} p \mathrm{~d} \zeta \\
& =P_{M}^{-}(x)-\int_{0}^{x_{S}} \int_{p_{\zeta}(\zeta)}^{(\zeta, x)} \nu(\zeta, p, x) \mathrm{d} p \mathrm{~d} \zeta
\end{aligned}
$$

where

$$
\bar{p}_{ \pm}(\zeta, x)=\left\{\begin{array}{cc} 
\pm \max \left( \pm x-\zeta, \zeta-x_{\mathrm{S}}\right) & (\zeta>0) \\
x & (\zeta=0)
\end{array}\right.
$$



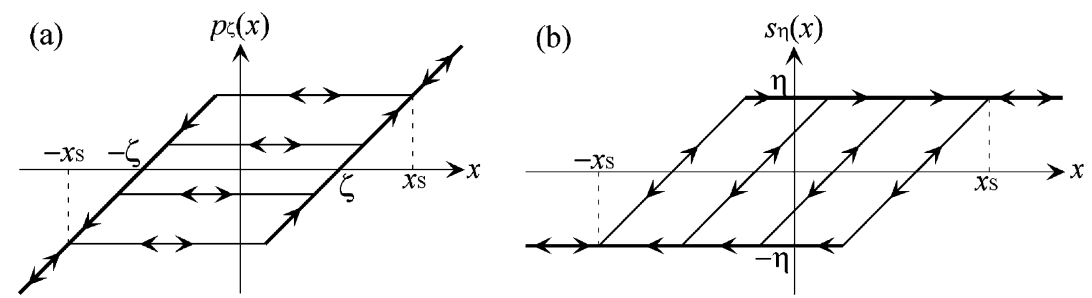

Fig. 1. Play and stop hysteron operators: (a) play hysteron given by (3) and (4), and (b) stop hysteron given by (14) and (15).

$$
P_{M}^{ \pm}(x)=\int_{0}^{x \mathrm{~s}} f_{\zeta}\left(\bar{p}_{ \pm}(\zeta, x), x\right) \mathrm{d} \zeta .
$$

Therein, $P_{\mathrm{M}}^{+}(x)$ and $P_{\mathrm{M}}^{-}(x)$ are the values of $P(x)$ on the ascending and descending curves of the major hysteresis loop, respectively. From (5), $P(x)$ is written as

$$
P(x)=P_{\mathrm{H}}(x)+\frac{\left\{P_{\mathrm{M}}^{+}(x)+P_{\mathrm{M}}^{-}(x)\right\}}{2}
$$

where

$P_{\mathrm{H}}(x)=\frac{1}{2} \int_{0}^{x_{\mathrm{S}}}\left[\int_{\bar{p}_{+}(\zeta, x)}^{p_{\zeta}(x)} \nu(\zeta, p, x) \mathrm{d} p-\int_{p_{\zeta}(x)}^{\bar{p}_{-}(\zeta, x)} \nu(\zeta, p, x) \mathrm{d} p\right] \mathrm{d} \zeta$.

Using the variable transformation $\alpha=p+\zeta$ and $\beta=p-\zeta$, $P_{\mathrm{H}}(x)$ is rewritten as

$$
P_{\mathrm{H}}(x)=\int_{x}^{x_{\mathrm{S}}} \int_{-x \mathrm{~s}}^{x} \mu(\alpha, \beta, x) \gamma_{\alpha \beta}(x) \mathrm{d} \beta \mathrm{d} \alpha
$$

where $\mu(\alpha, \beta, x)=\nu(\zeta, p, x) / 2$, and

$$
\gamma_{\alpha \beta}(x)=\left\{\begin{array}{cl}
\frac{1}{2} & \left(\frac{\alpha+\beta}{2} \leq p_{(\alpha-\beta) / 2}(x)\right) \\
-\frac{1}{2} & \left(\frac{\alpha+\beta}{2}>p_{(\alpha-\beta) / 2}(x)\right)
\end{array} .\right.
$$

Because this $\gamma_{\alpha \beta}(x)$ is identical to the elementary hysteresis operator of the Preisach model [9], the hysteresis model (8) is equivalent to the nonlinear (input-dependent) Preisach model proposed by Mayergoyz [4].

\section{B. Stop Model With Input-Dependent Shape Function}

A stop model [1]-[3] having an input-dependent shape function [8] is given as

$$
S(x)=\int_{0}^{x_{S}} g_{\eta}\left(s_{\eta}(x), x\right) \mathrm{d} \eta
$$

where $s_{\eta}$ is the stop hysteron operator having height $\eta$, and $g_{\eta}$ is the input-dependent shape function. $S(x)$ is assumed to become a single-valued function when $|x| \geq x_{\mathrm{S}}$. The stop hysteron operator of ordinary type is given by

$$
s_{\eta}(x)=\max \left(\min \left(x-x^{0}+s_{\eta}^{0}, \eta\right),-\eta\right)
$$

where $x^{0}$ and $s_{\eta}^{0}$ are the values of $x$ and $s_{\eta}$ at the previous timepoint. Because the function $S(x)$, given by (12) and (13), has a hysteretic property even when $|x| \geq x_{\mathrm{S}}$, this paper defines the operator $s_{\eta}(x)$ as

$$
\begin{aligned}
s_{\eta}(x) & =\left\{\begin{array}{cc}
\max \left(\min \left(x-x^{0 *}+\mathrm{s}_{\eta}^{0}, \eta\right),-\eta\right) & \left(\eta<x_{\mathrm{S}}\right) \\
x & \left(\eta=x_{\mathrm{S}}\right)
\end{array}\right. \\
x^{0 *} & =\max \left(\min \left(x^{0}, x_{\mathrm{S}}\right),-x_{\mathrm{S}}\right) .
\end{aligned}
$$

Fig. 1(b) illustrates characteristics of this operator for $\eta<x_{\mathrm{S}}$.

It holds that $s_{\xi}(x)+p_{\xi}(x)=x$ when $s_{\xi}^{0}+p_{\xi}^{0}=x^{0}$ [2]. For that reason, the stop model (12) can be rewritten as

$$
S(x)=\int_{0}^{x \mathrm{~s}} g_{\eta}\left(x-p_{\eta}(x), x\right) \mathrm{d} \eta .
$$

By setting $f_{\eta}\left(p_{\eta}(x), x\right)=g_{\eta}\left(x-p_{\eta}(x), x\right)$, the stop model (12) is represented by the play model (1). These two models are equivalent because the stop model also represents the play model in the same way.

Mayergoyz [4] proved a representation theorem for the nonlinear Preisach model. It shows that the wiping-out property and the property of equal vertical chords constitute the necessary and sufficient conditions for the representation of hysteretic nonlinearity by the nonlinear Preisach model. The same representation theorem holds for play and stop models having input-dependent shape functions because of the equivalence of the three models.

\section{Stop Model With Input-Independent Shape Function}

A stop model having an input-independent shape function is given as

$$
S_{0}(x)=\int_{0}^{x \mathrm{~s}} g_{\eta 0}\left(s_{\eta}(x)\right) \mathrm{d} \eta
$$

where $g_{\eta 0}$ is the input-independent shape function. This stop model has the property of equal vertical chords regardless of dc bias. Let $h^{+}(a, x)$ and $h^{-}(a, x)$ be the values of $S_{0}(x)$ on ascending and descending curves, respectively, for a back-andforth input variation $x=u+x_{0}(-a \leq u \leq a)$, where $a(0 \leq$ $\left.a \leq x_{\mathrm{S}}\right)$ is the amplitude and $x_{0}\left(\left|x_{0}\right| \leq\left|x_{\mathrm{S}}-a\right|\right)$ is an arbitrary $\mathrm{dc}$ bias. The input $x$ is assumed to have no extremum other than $x_{0} \pm a$. A previous work [5], [6] has shown that the vertical difference $\Delta h$ between $h^{ \pm}\left(a, u+x_{0}\right)$ does not depend on $x_{0}$

$$
h^{+}\left(a, u+x_{0}\right)-h^{-}\left(a, u+x_{0}\right)=\Delta h(a, u) .
$$

In other words, back-and-forth input variations of the same amplitude produce equal vertical chords regardless of the dc bias. 

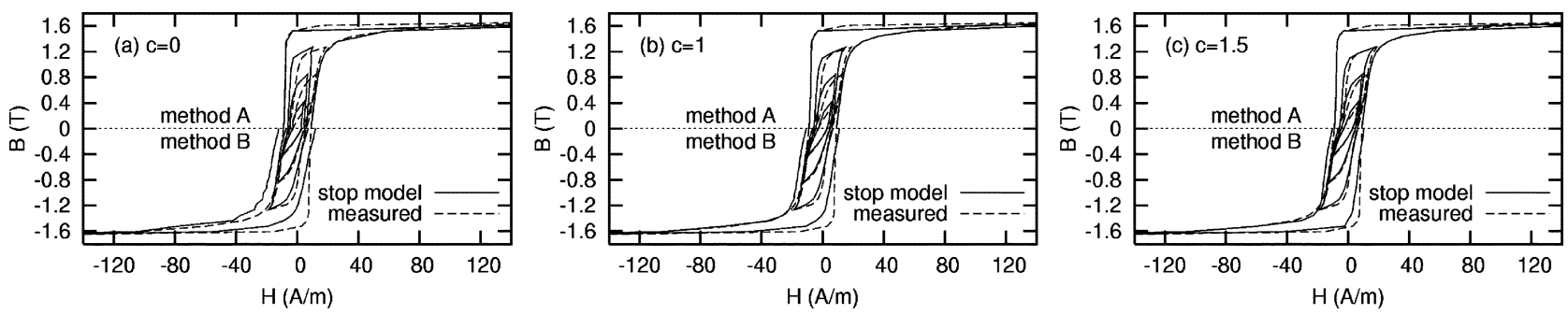

Fig. 2. $B-H$ loops of silicon steel sheet along the rolling direction.

A representation theorem holds for this stop model. It holds that (i) the property of equal vertical chords regardless of a dc bias, and (ii) the wiping-out property are the necessary and sufficient conditions for representation by the stop model having the input-independent shape function. The proof is given roughly as follows in a similar way [4] to the nonlinear Preisach model.

A given hysteretic function $H(x)$ is assumed to have properties (i) and (ii). The stop model $S_{0}(x)$ with input-independent shape function is assumed to have the same vertical chords $\Delta h(a, u)$ as $H(x)$ does. The major loop of $S_{0}(x)$ is presumed to coincide with that of $H(x)$. If it does not, $S_{0}(x)$ can be modified to have the same major loop as $H(x)$ as follows. The ascending and descending curves of the major loops of $H(x)$ and $S_{0}(x)$ are denoted by $H_{\mathrm{M}}^{ \pm}(x)$ and $S_{0 \mathrm{M}}^{ \pm}(x)$, respectively, where $H_{\mathrm{M}}^{+}(x)-H_{\mathrm{M}}^{-}(x)=S_{0 \mathrm{M}}^{+}(x)-S_{0 \mathrm{M}}^{-}(x)=\Delta h\left(x_{\mathrm{S}}, x\right)$. By replacing $S_{0}(x)$ by $S_{0}(x)+H_{\mathrm{M}}^{-}(x)-S_{0 \mathrm{M}}^{-}(x)$, the stop model can have the same major loop as $H(x)$.

For the sufficiency of properties (i) and (ii), it is shown that any high-order reversal curves of $H(x)$ coincide with those of $S_{0}(x)$ as follows. Let the $(2 k-1)$ th reversal curve of $H(x)$ from the negative saturation be $H_{2 k-1}(x)$, which results from the sequence of local input extrema $m_{1}\left(=-x_{\mathrm{S}}\right), m_{2}, \ldots, m_{2 k}$. The local minima $m_{2 i-1}$ and maxima $m_{2 i}(i=1, \ldots, k)$ can be assumed to be

$$
m_{1}<m_{3}<\ldots<m_{2 k-1}<m_{2 k}<m_{2 k-2}<\ldots<m_{4}<m_{2}
$$

because other local extrema can be ignored because of the wiping-out property. Then $H_{2 k-1}(x)$ is uniquely given as

$$
\begin{array}{r}
H_{2 k-1}(x)=H_{M}^{+}(x)-\sum_{i=1}^{k} \Delta h\left(\frac{m_{2 i}-m_{2 i-1}}{2}, x-\frac{m_{2 i-1}+m_{2 i}}{2}\right) \\
+\sum_{i=1}^{k-1} \Delta h\left(\frac{m_{2 i}-m_{2 i+1}}{2}, x-\frac{m_{2 i}+m_{2 i+1}}{2}\right) \cdot
\end{array}
$$

This coincides with the reversal curve of $S_{0}(x)$ having the same local input extrema sequence. Similarly, the $2 k$ th reversal curve of $H(x)$ coincides with that of $S_{0}(x)$.

The necessity of properties (i) and (ii) seems obvious.

\section{IMPROVEMENT OF THE STOP MODEL REPRESENTATION ACCURACY}

\section{A. Weighting Function}

The input-dependent shape function

$$
g_{\eta}(s, x)=w(x) g_{\eta 0}(s)
$$

was introduced in [8], where $w(x)$ is called the weighting function. Equations (12) and (21) imply that $S(x) / w(x)$ represents the stop model (17) with input-independent shape function. Therefore, for a hysteretic function $H(x)$ to be represented accurately by the stop model using (21), $H(x) / w(x)$ should have the property of equal vertical chords regardless of $\mathrm{dc}$ bias.

A simple weighting function (22) was proposed in [8]

$$
w(x)=\Delta h\left(x_{\mathrm{S}}, x\right)=H_{\mathrm{M}}^{+}(x)-H_{\mathrm{M}}^{-}(x) .
$$

This paper examines the weighting function in the following form:

$$
w(x)=\Delta h\left(x_{\mathrm{S}}, 0\right)\left\{\frac{\Delta h\left(x_{\mathrm{S}}, x\right)}{\Delta h\left(x_{\mathrm{S}}, 0\right)}\right\}^{c}
$$

where $c$ is a constant. This weighting function is identical to that of (22) when $c=1$, whereas the shape function $g_{\eta}$ becomes input-independent when $c=0$.

\section{B. Representation of Hysteretic Characteristics of Grain-Oriented Silicon Steel Sheet Along the Rolling Direction}

DC $B-H$ loops of a grain-oriented silicon steel sheet (JIS: 35p105) are represented by the stop model using the weighting function (23). Two identification methods proposed in [5], [6], and [8] are used; the former one (called "the method A" in this paper) uses the widths of $B-H$ loops and the latter ("the method B") uses the descending curves of symmetric $B-H$ loops.

Fig. 2 shows symmetric $B-H$ loops of the steel sheet along its rolling direction, where 20 symmetric $B-H$ loops are used for identification. Fig. 3 compares the vertical chords $\Delta h / w(B)$ of measured symmetric and asymmetric loops of the steel sheet, where $B_{0}$ and $B_{\mathrm{S}}$ are the dc bias of input $B$ and the saturation magnetic flux density, respectively, corresponding to $x_{0}$ and $x_{\mathrm{S}}$. The dc bias for the asymmetric loops is given by $B_{0}=-B_{\mathrm{S}}+a$, where $a$ is the amplitude of input. The parameter $c=1.5$ is used to roughly minimize the difference of vertical chords between symmetric and asymmetric loops.

A large discrepancy of vertical chords is seen in Fig. 3(a) between the symmetric and asymmetric loops when $c=0$.

Fig. 2(a) shows that this strong dependency of vertical chords on the dc bias causes a discrepancy between the measured and simulated loops. On the other hand, Fig. 3(c) shows that the weighting function with $c=1.5$ reduces the difference of vertical chords effectively. Fig. 2(c) shows that the weighting 

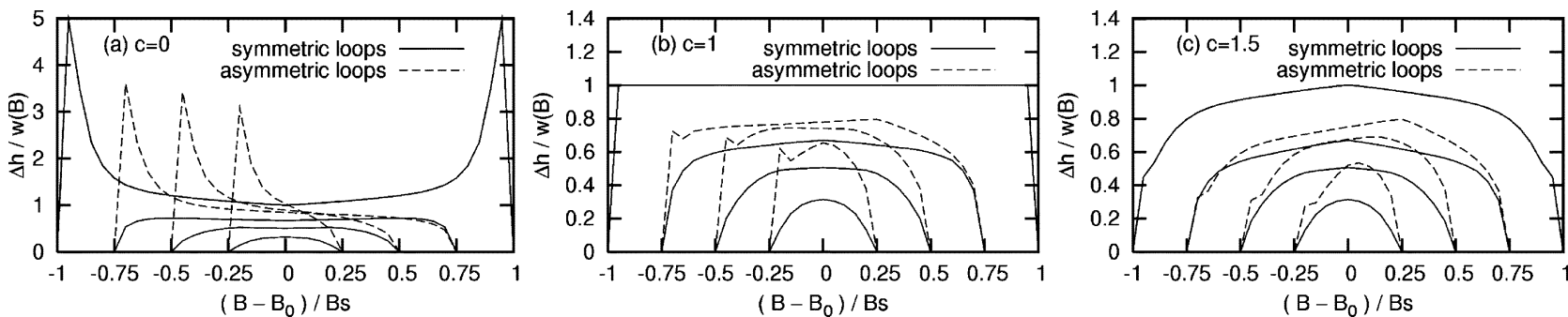

Fig. 3. Vertical chords $\Delta h / w$ of silicon steel sheet along the rolling direction.
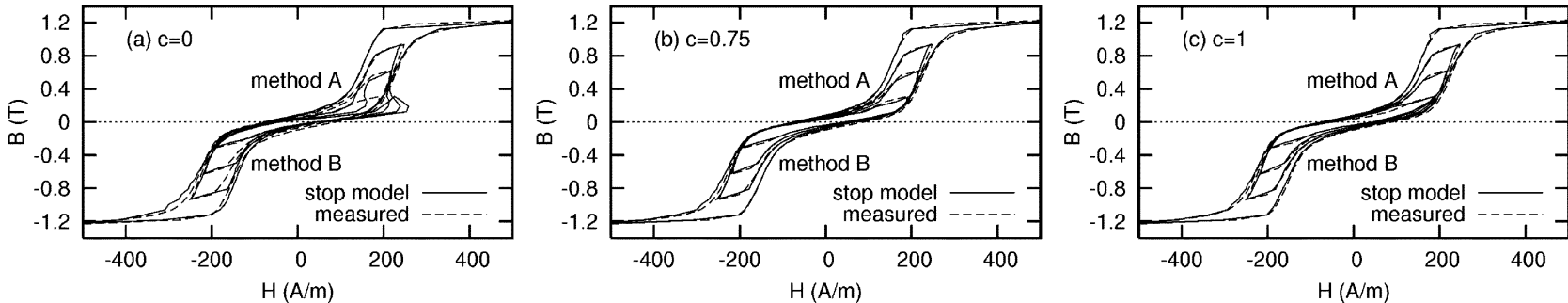

Fig. 4. $B-H$ loops of silicon steel sheet along the transverse direction.

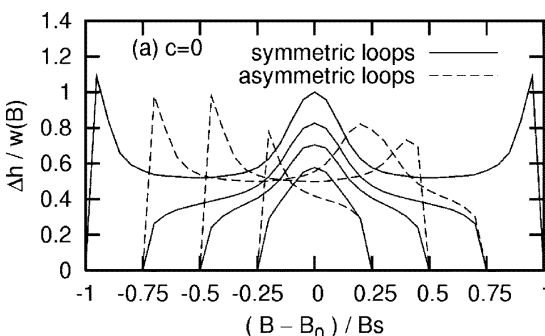

$\left(\mathrm{B}-\mathrm{B}_{0}\right) / \mathrm{Bs}$

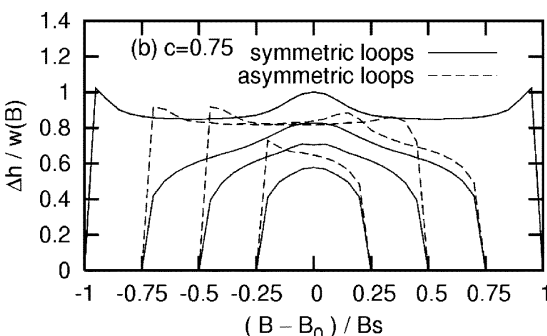

$\left(\mathrm{B}-\mathrm{B}_{0}\right) / \mathrm{Bs}$

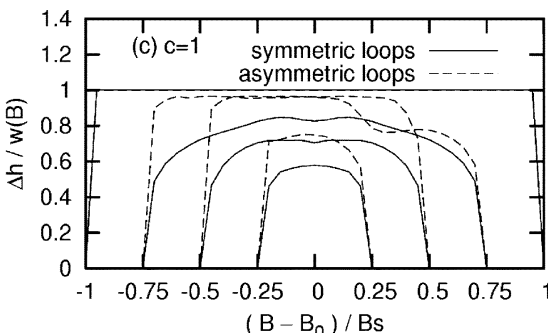

$\left(\mathrm{B}-\mathrm{B}_{0}\right) / \mathrm{Bs}$

Fig. 5. Vertical chords $\Delta h / w$ of silicon steel sheet along the transverse direction.

function improves the representation accuracy of $B-H$ loops effectively for both identification methods.

\section{Representation of Hysteretic Characteristics of a Grain- Oriented Silicon Steel Sheet Along the Transverse Direction}

Fig. 4 shows simulated symmetric $B-H$ loops of the grain-oriented silicon steel sheet along the transverse direction. Fig. 5 compares the vertical chords of symmetric and asymmetric loops. The strong dependency of vertical chords on the dc bias is seen in Fig. 5(a) for $c=0$, which causes a large representation error of $B-H$ loops, as shown in Fig. 4(a). Fig. 5(b) and (c) shows that the weighting functions with $c=0.75$ and 1 reduce the difference of vertical chords. Fig. 4(b) and (c) shows that appropriate weighting functions reduce the representation error effectively for both identification methods.

\section{CONCLUSION}

This paper derives a representation theorem for the stop and play models with input-dependent shape functions from the equivalence of these models to the nonlinear Preisach model. A representation theorem for the stop model having an input-independent shape function is also given. The latter theorem can be used to determine an appropriate weighting function for the input-dependent shape function of the stop model.

\section{ACKNOWLEDGMENT}

This work was supported in part by the Japan Society for the Promotion of Science, Grant-in-Aid for Scientific Research (C), 16560243.

\section{REFERENCES}

[1] M. A. Krasnosel'skii and A. V. Pokrovskii, Systems With Hysteresis. Berlin, Germany: Springer-Verlag, 1989.

[2] A. Visintin, Differential Models of Hysteresis. Berlin, Germany: Springer-Verlag, 1994

[3] S. Bobbio, G. Miano, C. Serpico, and C. Visone, "Models of magnetic hysteresis based on play and stop hysterons," IEEE Trans. Magn., vol 33, no. 6, pp. 4417-4426, Nov. 1997.

[4] I. D. Mayergoyz, Mathematical Models of Hysteresis. New York: Springer-Verlag, 1991.

[5] T. Matsuo and M. Shimasaki, "Isotropic vector hysteresis represented by superposition of stop hysteron models," IEEE Trans. Magn., vol. 37, no. 5, pp. 3357-3361, Sep. 2001.

[6] — , "Correction to: 'Isotropic vector hysteresis represented by superposition of stop hysteron models'," IEEE Trans. Magn., vol. 37, no. 6 , p. 3995 , Nov. 2001

[7] T. Matsuo, D. Shimode, Y. Terada, and M. Shimasaki, "Application of stop and play models to the representation of magnetic characteristics of silicon steel sheet," IEEE Trans. Magn., vol. 39, no. 3, pp. 1361-1364, May 2003

[8] T. Matsuo, Y. Terada, and M. Shimasaki, "Stop model with input-dependent shape function and its identification methods," IEEE Trans. Magn., vol. 40, no. 4, pp. 1776-1783, Jul. 2004.

[9] M. Brokate, "Some mathematical properties of the Preisach model for hysteresis," IEEE Trans. Magn., vol. 25, no. 4, pp. 2922-2924, Jul. 1989.

Manuscript received June 8, 2004. 